\title{
O RUÍDO GERADO DURANTE A MANIPULAÇÃO DAS INCUBADORAS: IMPLICAÇÕES PARA O CUIDADO DE ENFERMAGEM ${ }^{1}$
}

\author{
Milena D. Oliveira Rodarte ${ }^{2}$ \\ Carmen Gracinda Silvan Scochi ${ }^{3}$ \\ Adriana Moraes Leite ${ }^{3}$ \\ Cristina Ide Fujinaga \\ Nelma Ellen Zamberlan ${ }^{5}$ \\ Thaila Correa Castra $f^{6}$
}

Rodarte MDO, Scochi CGS, Leite AM, Fujinaga Cl, Zamberlan NE, Castral TC. O ruído gerado durante a manipulação das incubadoras: implicações para o cuidado de enfermagem. Rev Latino-am Enfermagem 2005 janeiro-fevereiro; 13(1):79-85.

Estudo tipo experimento não controlado. Objetivo: quantificar o ruído de impacto das 23 incubadoras em operação das unidades de cuidado intensivo e intermediário de um hospital universitário, público, de Ribeirão Preto-SP. Metodologia: medições ocorreram durante a manipulação das incubadoras nos modos cuidadoso e brusco, visando ao ruído de impacto gerado pelas diversas situações de manipulações com a incubadora. Resultados: praticamente em todas situações de manipulação, tanto no modo cuidadoso como no brusco, o grupo $B$ apresentou NPS mais elevados, seguido pelos grupos A e D. O grupo C foi o que demonstrou os menores NPS durante as diversas situações de manipulação. Mesmo a manipulação cuidadosa gerou forte nível de ruído e quase sempre é dobrado ou triplicado o NPS quando a mesma situação é realizada bruscamente. A manipulação cuidadosa, juntamente com menor tempo de uso da incubadora, mostrou-se eficaz na redução do ruído produzido durante o manuseio do equipamento.

DESCRITORES: ruído; controle de ruídos; medição de ruído; fonoaudiologia; enfermagem neonatal

\section{NOISE GENERATED DURING INCUBATOR MANIPULATION: IMPLICATIONS FOR NURSING CARE}

Uncontrolled experimental study. Aim: quantify the impact noise of the 23 incubators functioning in the intensive and intermediate care units of a public university hospital in Ribeirão Preto-SP. Methodology: measuring occurred during incubator manipulation in a careful and in a rough way, considering the impact noise generated by the various situations in which the incubator was manipulated. Results: In practically all manipulation situations in both the careful and the rough way, group B presented higher NPS, followed by group A and D. Group C demonstrated the lowest NPS during the different manipulation situations. Even careful manipulation generated a strong noise level, and NPS almost always doubled or tripled when the same situation was realized roughly. Careful manipulation, together with lower incubator use time, revealed to be effective in reducing the noise produced during equipment manipulation.

DESCRIPTORS: noise; noise control; noise measurement; speech, language and hearing sciences; neonatal nursing

\section{EL RUIDO GENERADO DURANTE EL MANEJO DE INCUBADORAS: IMPLICACIONES PARA LA ATENCIÓN DE ENFERMERÍA}

Estudio del tipo experimento no controlado. Objetivo: cuantificar el ruido de impacto de las 23 incubadoras en operación de las unidades de cuidado intensivo e intermedio de un hospital universitario público de Ribeirão Preto-SP. Metodología: mediciones ocurrieron durante el manejo de las incubadoras de modo cuidadoso y brusco, visando el ruido de impacto generado por las diversas situaciones de manejo con la incubadora. Resultados: En prácticamente todas las situaciones de manejo, tanto de modo cuidadoso como de modo brusco, el grupo B presentó NPS más elevados, seguido por el grupo A y D. El grupo C fue el que demostró los menores NPS durante las diversas situaciones de manejo. Mismo el manejo cuidadoso generó fuerte nivel de ruido, y el NPS casi siempre se dobla o triplica cuando la misma situación se realiza bruscamente. El manejo cuidadoso, en conjunto con menor tempo de uso de la incubadora, se mostró eficaz en la reducción del ruido producido al manosear el equipo.

DESCRIPTORES: ruido; control del ruido; medición del ruido; fonoaudiología; enfermería neonatal

\footnotetext{
${ }^{1}$ Trabalho realizado no Hospital das Clínicas da Faculdade de Medicina de Ribeirão Preto da Universidade de São Paulo junto ao Departamento de Ginecologia e Obstetrícia; ${ }^{2}$ Fonoaudióloga, Mestre pelo Programa de Pós-Graduação; ${ }^{3}$ Enfermeira, Docente, e-mail: cscochi@eerp.usp.br; ${ }^{4}$ Fonoaudióloga, Mestre e Doutoranda pelo Programa de Pós-Graduação. Escola de Enfermagem de Ribeirão Preto, da Universidade de São Paulo, Centro Colaborador da OMS para o desenvolvimento da pesquisa em enfermagem; ${ }^{5}$ Fonoaudióloga, Aprimoramento Profissional em Fonoaudiologia do Hospital das Clínicas da Faculdade de Medicina de Ribeirão Preto da Universidade de São Paulo; ${ }^{6}$ Aluna do $7^{\circ}$ semestre do Curso de Graduação em Enfermagem da Escola de Enfermagem de Ribeirão Preto, da Universidade de São Paulo, Centro Colaborador da OMS para o desenvolvimento da pesquisa em enfermagem, Bolsista de Iniciação Científica PIBIC/USP/CNPq 2003/2004
} 
O ruído gerado durante a manipulação...

Rodarte MDO, Scochi CGS, Leite AM, Fujinaga Cl, Zamberlan NE, Castral TC.

\section{INTRODUÇÃO}

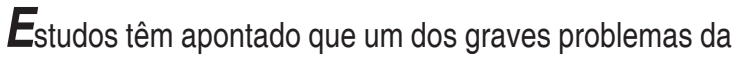
unidade de terapia intensiva neonatal (UTIN) é o ambiente superestimulante comprometendo o processo de desenvolvimento e crescimento, devido ao fato de, nos recém-nascidos (RNs), em especial nos prematuros, os receptores sensoriais serem extremamente sensíveis ao ambiente ${ }^{(1-2)}$. Assim, um dos problemas relevantes é a poluição sonora intensa que se encontra constantemente presente nesse local, decorrente de diversas fontes, tais como a circulação de pessoas na unidade, dos equipamentos de suporte à vida, como respiradores mecânicos, berços aquecidos, aparelhos de fototerapia, bombas de infusão, monitores cardiorespiratórios e de temperatura cutânea, incubadoras, vozes, alarmes, rádios, dentre outros, predispondo a criança aos danos auditivos e às alterações fisiológicas e comportamentais ${ }^{(2)}$.

Apesar da diminuição da mortalidade entre os recémnascidos pré-termo e de baixo peso ao nascer, essas crianças continuam sendo de risco, apresentando maior incidência de problemas motores, sensoriais (tais como os problemas auditivos) e outros problemas do desenvolvimento, que podem ser, em parte, atribuídos aos cuidados recebidos na UTIN ${ }^{(2-3)}$. Com o aumento da sobrevida dos prematuros, houve um conseqüente aumento de RNs com risco para a perda auditiva.

0 cérebro do bebê prematuro encontra-se imaturo para processar e registrar as informações sensoriais, sendo extremamente sensitivo e incapaz de selecionar as informações recebidas devido à falta dos controles inibitórios ${ }^{(3)}$. Além disso, os prematuros são mais susceptíveis aos efeitos do ambiente, sendo que, quanto menor a idade gestacional, maior o comprometimento, pois o desenvolvimento cerebral, não estando completo, aumenta o risco de maturação cerebral anormal $^{(1)}$.

As respostas fisiológicas e comportamentais do $\mathrm{RN}$ ao ruído incluem: hipóxia; aumento da pressão intracraniana e da pressão sangüínea, predispondo a hemorragia craniana intraventricular nos prematuros; apnéia; bradicardia; apagamento comportamental ou isolamento da interação social; alteração do estado de repouso e sono, levando à fadiga, agitação, irritabilidade e choro; aumento do consumo do oxigênio e da freqüência cardíaca, resultando no aumento do consumo calórico e, conseqüentemente, ganho de peso lento. Além
Rev Latino-am Enfermagem 2005 janeiro-fevereiro; 13(1):79-85 www.eerp.usp.br/rlae disso, os níveis de ruído podem induzir a uma redução global na resposta auditiva e no desenvolvimento ${ }^{(1)}$.

Nas unidades neonatais de alta complexidade, como explicitado anteriormente, os bebês estão expostos a várias fontes de ruído ambiental, as quais podem potencializar os riscos para a deficiência auditiva. Muitas dessas fontes de ruído são decorrentes do próprio processo terapêutico, como o uso de drogas ototóxicas e equipamentos de suporte à vida. Em neonatos, ainda não foi determinado um critério de proteção sobre os efeitos do ruído, não sendo ainda estabelecido o nível de decibéis $(\mathrm{dB})^{\star}$ que provoca o dano auditivo. Entretanto, há indícios de que o sistema auditivo do prematuro, pela sua própria imaturidade global, é provavelmente mais susceptível às lesões que podem ser causadas por um ambiente super estimulante auditivamente, devido à combinação do ruído com o uso de medicações ototóxicas, que aumentam ainda mais o risco.

A poluição sonora nas UTIN faz-se presente diariamente, ocorrendo tanto ruídos graves como agudos, ultrapassando intensidades de $110 \mathrm{~dB}$, nefastos à cóclea do ouvido humano, não sendo prejudiciais somente ao adulto que fica exposto a eles $08,10 \mathrm{ou}$ 12 horas, mas muito mais nocivos ao bebê de alto risco, o qual fica exposto a esse ambiente ruidoso continuamente, durante vários dias ou até meses ${ }^{(6)}$.

A preocupação com os níveis de ruído em incubadoras data da década de 70. No Brasil, somente na década de 90 é que tiveram início os estudos direcionados à questão do ruído em ambiente neonatal, especificamente em incubadoras. Nessa ocasião, não havia uma legislação específica que determinasse o nível de ruído permitido em incubadoras, existindo, no momento, somente a norma da American Academy of Pediatrics.

De acordo com o US Envinonmental Protection Agency, os níveis de ruído acima de $45 \mathrm{~dB} / \mathrm{A}$ devem ser evitados na UTIN e dentro das incubadoras ${ }^{(7)}$. A American Academy of Pediatrics sugere $58 \mathrm{~dB} / \mathrm{A}$ como o nível permitido de exposição de ruído ao $\mathrm{RN}^{(8)}$. A legislação brasileira, em 1997, passa a recomendar 60 dB/A como o máximo Nível de Pressão Sonora (NPS) ${ }^{\star *}$ permitido dentro da incubadora ${ }^{(9)}$. Estudos $^{(3-4,8,10-16)}$ têm apontado, tanto no ambiente neonatal quanto em incubadoras, níveis sonoros bem mais intensos que 0 estipulado pelas normas, principalmente durante o manuseio das incubadoras. Ressaltamos que os bebês que necessitam do uso da incubadora estão expostos tanto ao ruído do motor (ruído contínuo), quanto ao de sua manipulação(ruído de impacto).

\footnotetext{
* dB: decibéis é uma unidade de medida referenciada em escala logarítmica, definida em função da intensidade ou pressão sonora ${ }^{(4)}$. Escalas padronizadas internacionalmente foram criadas para a medição do ruído, sendo denominadas circuitos ou escalas de compensação $A(d B / A), B(d B / B)$ e $C$ (dB/C), e projetadas para reproduzirem a audibilidade em função da freqüência sonora ${ }^{(5)}$.

${ }^{\star *}$ NPS: processo audiológico de medida de intensidade sonora equivalente a $20 \mu \mathrm{Pa}$, menor pressão sonora audível ${ }^{(5)}$.
} 
Tendo a preocupação de promoção da saúde e da qualidade de vida das crianças nascidas pré-termo e de alto risco, com atenção especial à prevenção de danos auditivos, motivamo-nos a realizar a presente investigação, tendo como objeto o estudo dos níveis de ruído de impacto encontrados nas incubadoras de um hospital universitário de Ribeirão Preto-SP, por constituir-se em um equipamento freqüentemente utilizado na assistência ao prematuro, por vários dias ou meses.

É notado, também, que, na assistência a essa clientela, não há uma rotina específica para o manuseio da incubadora e que nem sempre os profissionais estão atentos à sua manipulação sutil visando, à prevenção de ruídos, além do que são raras as unidades neonatais que contam com serviços de manutenção técnica preventiva desse equipamento.

\section{MATERIAL E MÉTODO}

O estudo do tipo experimento não controlado foi desenvolvido com as incubadoras do berçário de cuidados intermediários e da UTIN de um hospital-escola, público, de referência terciária na assistência perinatal e neonatal, que atende a clientela do município de Ribeirão Preto-SP, das regiões circunvizinhas e de outros estados. As incubadoras foram testadas nos períodos em que estavam desocupadas, sem a presença dos RNs.

Foi utilizado um decibelímetro digital (Minipa MSL-1352) para a mensuração dos NPSs das 23 incubadoras que estavam em regime rotineiro de operação, sendo que 16 se encontravam na unidade de cuidados intermediários e sala de estoque de equipamentos, e 7, na UTIN do hospital-escola.

O decibelímetro foi colocado no interior da incubadora, sobre um suporte com $10 \mathrm{~cm}$ de altura, o centro da plataforma de base, mantendo-se a temperatura de controle da incubadora entre $30^{\circ} \mathrm{Ce}$ $33^{\circ} \mathrm{C}$ e a umidade máxima (nas incubadoras que possuíam esse recurso), segundo as normas técnicas vigentes ${ }^{(9)}$.

As medidas ocorreram com a incubadora em funcionamento normal durante a manipulação do equipamento, visando ao ruído de impacto gerado pelas seguintes situações de manipulação nos modos brusco e cuidadoso: abertura e fechamento das portinholas do lado do painel de controle; no ajuste da plataforma de base, elevando-a para posicionamento no suporte e abaixando-a para posicionamento horizontal; no ato de retirar e colocar a plataforma de base; abertura e fechamento da porta de cuidado intensivo (nos modelos em que havia); quando se eleva e abaixa a cúpula da incubadora; abrindo e fechando as portas dos gabinetes inferiores (nos modelos em que havia); no ato de tamborilar sobre a cúpula de acrílico (com portinholas fechadas); quando se coloca a mamadeira sobre a cúpula (portinholas fechadas); quando se coloca o prontuário sobre a cúpula (portinholas fechadas) e quando se movimenta a incubadora (portinholas fechadas).

A medição do ruído de impacto foi realizada no circuito de compensação $\mathrm{C}$ e circuito de resposta rápida (FAST), indicada para as medidas de ruídos de impacto, de acordo com a NR 15, em seu Anexo № $2^{(17)}$. Foram coletadas três medidas do ruído para se obter uma média, respeitando 0 intervalo de trinta segundos entre cada medida. Em todas as medidas, a função MAX do aparelho foi ativada, fixando-se assim, no visor, o pico máximo do ruído atingido durante cada manipulação realizada. Todas as medidas foram coletadas por uma das pesquisadoras, e as situações de manipulação foram realizadas por uma única auxiliar de pesquisa. Não houve um controle sistemático para a variável força, com relação ao modo de realização cuidadoso e brusco, sendo usado em relação a essa variável o referencial subjetivo que a auxiliar de pesquisa tinha para as manipulações. Essa variável é considerada um erro não aleatório, não se podendo, portanto, controlá-la, pois depende do indivíduo. 0 ambiente de mensuração foi sempre o mesmo: uma sala silenciosa afastada do fluxo rotineiro do ambiente neonatal.

As 23 incubadoras testadas foram divididas em quatro grupos diferentes. O grupo A foi composto por 8 incubadoras da mesma marca e modelo, com o período de uso de 15 a 16 anos e procedência nacional; o grupo B, por 8 incubadoras nacionais da mesma marca $e$ modelo, com o período de uso entre 16 a 18 anos; o $\mathrm{C}$ foi composto por 4 incubadoras, sendo 3 da mesma marca e modelo e de procedência estrangeira e 1 nacional de outra marca e modelo, com o período de uso entre 4 e 5 anos, e 0 grupo D, por 3 incubadoras, sendo 2 estrangeiras da mesma marca e modelo e 1 nacional de outra marca e modelo, com o período de uso de 4 a 6 anos. Para essa conformação dos grupos, além do período de uso, priorizamos as incubadoras a serem testadas nas mesmas situações de manipulação. 


\section{RESULTADOS}

Tabela 1 - Distribuição dos níveis médios de ruído de impacto (dB/C NPS), segundo os grupos A, B e C nos modos cuidadoso e brusco de manipulação

\begin{tabular}{|c|c|c|c|c|c|c|c|c|c|c|c|c|c|c|c|}
\hline \multirow{3}{*}{$\begin{array}{l}\text { SITUAÇÕES DE } \\
\text { MANIPULAÇÃO }\end{array}$} & \multirow{2}{*}{\multicolumn{3}{|c|}{ A }} & \multirow{2}{*}{\multicolumn{6}{|c|}{ GRUPOS }} & \multirow{2}{*}{\multicolumn{3}{|c|}{ D }} & \multirow{2}{*}{\multicolumn{3}{|c|}{ GERAL }} \\
\hline & & & & & & & & & & & & & & & \\
\hline & $\mu_{c}$ & $\mu_{\text {в }}$ & Dif & $\mu c$ & $\mu_{\text {в }}$ & Dif & $\mu_{c}$ & $\mu_{\text {в }}$ & Dif & $\mu c$ & $\mu_{\mathrm{B}}$ & Dif & $\mu c$ & $\mu_{\text {в }}$ & Dif \\
\hline Abrir portinholas & 82,3 & 87,5 & 5,2 & 90,9 & 91,9 & 1,0 & 70,3 & 78,4 & 8,0 & 75,8 & 77,0 & 1,1 & 82,3 & 86,1 & 3,8 \\
\hline Fechar portinholas & 86,3 & 94,5 & 8,1 & 93,3 & 98,4 & 5,2 & 71,5 & 92,9 & 21,4 & 78,8 & 96,4 & 17,6 & 85,2 & 95,8 & 10,5 \\
\hline Elevar plataforma base & 79,2 & 92,4 & 13,2 & 77,2 & 95,4 & 18,3 & 74,1 & 83,2 & 9,1 & 82,3 & 84,7 & 2,5 & 78,1 & 90,9 & 12,9 \\
\hline Abaixar plataforma base & 78,7 & 93,3 & 14,5 & 78,6 & 95,2 & 16,6 & 76,7 & 87,6 & 10,9 & 82,4 & 87,6 & 5,2 & 78,8 & 92,2 & 13,4 \\
\hline Retirar plataforma base & 76,3 & 88,7 & 12,3 & * & * & * & * & * & * & * & * & * & 76,3 & 88,7 & 12,3 \\
\hline Colocar plataforma base & 77,7 & 92,1 & 14,4 & * & * & * & * & * & * & * & * & * & 77,7 & 92,1 & 14,4 \\
\hline Abrir porta cuidado intensivo & 79,8 & 89,0 & 9,2 & * & * & * & 78,0 & 81,9 & 3,9 & 78,6 & 83,7 & 5,0 & 79,2 & 86,2 & 7,1 \\
\hline Fechar porta cuidado intensivo & 88,6 & 97,0 & 8,4 & * & * & * & 76,3 & 88,1 & 11,8 & 77,5 & 91,1 & 13,6 & 83,4 & 93,7 & 10,2 \\
\hline Elevar cúpula & 83,3 & 89,0 & 5,7 & 81,9 & 86,4 & 4,5 & 81,6 & 88,6 & 7,1 & 84,6 & 88,5 & 4,0 & 82,7 & 88,0 & 5,3 \\
\hline Abaixar cúpula & 86,4 & 97,0 & 10,6 & 86,1 & 95,6 & 9,5 & 84,4 & 93,4 & 9,0 & 87,4 & 94,6 & 7,2 & 86,1 & 95,6 & 9,5 \\
\hline Abrir porta gabinete inferior & 77,8 & 80,7 & 3,0 & 77,2 & 79,7 & 2,5 & * & * & * & 83,2 & 85,9 & 2,7 & 78,4 & 81,1 & 2,7 \\
\hline Fechar porta gabinete inferior & 79,8 & 89,9 & 10,1 & 80,0 & 92,4 & 12,4 & * & * & * & 81,8 & 93,3 & 11,5 & 80,2 & 91,4 & 11,2 \\
\hline Tamborilar cúpula & 82,6 & 92,4 & 9,8 & 87,0 & 93,9 & 7,0 & 76,1 & 87,0 & 10,9 & 79,2 & 89,3 & 10,1 & 82,6 & 91,6 & 9,1 \\
\hline Colocar mamadeira cúpula & 77,6 & 94,1 & 16,5 & 80,3 & 96,5 & 16,2 & 72,3 & 92,1 & 19,9 & 74,1 & 91,5 & 17,5 & 77,2 & 94,3 & 17,1 \\
\hline Colocar prontuário cúpula & 72,5 & 91,4 & 18,8 & 75,3 & 96,3 & 21,0 & 68,7 & 84,6 & 15,9 & 72,6 & 92,0 & 19,4 & 72,8 & 92,0 & 19,2 \\
\hline Empurrar incubadora & 79,9 & 85,2 & 5,3 & 79,4 & 84,4 & 5,1 & 76,4 & 83,2 & 6,8 & 81,6 & 88,1 & 6,5 & 79,4 & 85,0 & 5,6 \\
\hline
\end{tabular}

$\mu_{C}$ - média no modo cuidadoso de manipulação

$\mu_{\mathrm{B}}$ - média no modo brusco de manipulação

Dif - diferença entre $\mu_{\mathrm{B}}$ e $\mu_{\mathrm{C}}$

* Neste determinado grupo de incubadoras não foram realizadas estas situações de manipulação

A medida estatística de posição (média aritmética) usada na apresentação dos dados foi adequada para a análise. Os desviospadrão (variabilidade dos valores em torno da média) encontrados para todas as médias foram de 0,3 a 9,4 , sendo considerados valores pequenos, demonstrando, portanto, que essas variáveis são significativas.

A análise das possíveis diferenças com relação à força empregada pela auxiliar de pesquisa foi realizada em um teste não paramétrico, com nível de significância $\mu=0,05$. Os resultados estatísticos obtidos foram: grupo $A, p=0,08$; grupo $B, p=0,785$; grupo $C, p=0,366$ e grupo $D, p=0,570$, constatando-se, portanto, que não houve diferença estatisticamente significante entre a força empregada.

\section{DISCUSSÃO}

Em praticamente todas as situações de manipulação, tanto no modo cuidadoso como no brusco de realização, o grupo B apresentou os NPSs mais elevados, seguido pelo grupo A e D. 0 grupo $\mathrm{C}$ foi o que demonstrou os menores NPSs durante as diversas situações de manipulação. É relevante relembrar aqui que o NPS é um processo relativo de medida de intensidade, que tem como unidade de medida o decibel, que é uma escala logarítmica, portanto, quando 0 nível de pressão sonora é duplicada, o NPS aumenta em apenas 6 $\mathrm{dB}^{(18)}$.

No grupo A, durante a abertura das portinholas, observamos que mesmo a manipulação cuidadosa gerou um intenso nível de ruído e que este quase teve o NPS dobrado no modo brusco. $O$ grupo B foi o que produziu maior média de intensidade sonora na situação de abertura e fechamento das portinholas e quase não apresentou diferença em dB nos diferentes modos de manipulação, fato que se justifica, pois, nesse grupo, os equipamentos possuem de 16 a 18 anos de uso.

As médias dos grupos $\mathrm{C}$ e $\mathrm{D}$ apresentaram valores próximos na abertura das portinholas. Nesses dois grupos, as médias apresentaram NPSs muito inferiores tanto no modo brusco como no cuidadoso, possivelmente pelos equipamentos terem somente 4 a 6 anos de uso.

$\mathrm{Na}$ situação de fechar as portinholas, os grupos A e B apresentaram níveis sonoros bem elevados para essa mesma situação. As incubadoras dos grupos $\mathrm{C}$ e D apresentaram, durante o fechamento das portinholas, no modo cuidadoso de manipulação, NPSs significativamente inferiores aos dos outros dois grupos; já no modo brusco, os valores ficaram próximos. Nesses grupos, evidenciamos a importância da manipulação cuidadosa para redução do nível de ruído produzido, durante essa constante situação de manipulação, pois constatamos níveis de diferença entre o modo brusco e cuidadoso de até $21,4 \mathrm{~dB} / \mathrm{C}$ NPS (Grupo C), diferença de mais que o quádruplo de ruído produzido entre um modo e o outro.

A maioria das incubadoras em uso nos hospitais é antiga, sendo necessário bater as portinholas do equipamento para poder fechá-las ${ }^{(10)}$. Esse fato também foi observado na instituição onde realizamos a coleta dos dados, com as incubadoras dos grupos $\mathrm{B}$ e A, que eram de modelos mais antigos.

Outros estudos também ressaltam níveis de ruído intensos durante a abertura das portinholas: média de $112 \mathrm{~dB}$ NPS, em pico $^{(11)}$; 76 a 94 dB (linear) NPS ${ }^{(8)}$; média de 96,2 dB/F NPS ${ }^{(4)} ; 72,3$ dB NPS, 
ao abrir as portinholas no modo suave, e $81 \mathrm{~dB}$ NPS, no modo brusco $^{(12) ;} 85$ a 95 dB/A NPS ${ }^{(13)}$. Ao fechar as portinholas, também são constatados níveis intensos: pico de 124,5 dB NPS, elevando-se para $126,5 \mathrm{~dB}$ NPS, se fechadas de modo brusco ${ }^{(11)}$; 81 a $126 \mathrm{~dB}$ (linear) $\mathrm{NPS}^{(8)}$; média de $107 \mathrm{~dB} / \mathrm{F} \mathrm{NPS}^{(4)}$; 72 dB NPS, no modo suave, e 83,3 dB NPS, no modo brusco ${ }^{(11)} ; 85$ a 95 dB/A NPS ${ }^{(13)} ; 108 \mathrm{~dB}^{(14)}$ e pico de $111 \mathrm{~dB} /$ A NPS $^{(15)}$.

Ressaltamos que a comparação dos resultados com outros autores é limitada, pois alguns não especificaram qual escala de compensação foi usada nessas medições, e outros usaram circuitos de compensação inadequados para a mensuração de ruídos de impacto, sendo recomendadas as escalas linear e $C^{(17)}$.

As incubadoras dos grupos A e B apresentam, em suas plataformas de base, peças e suportes metálicos, o que contribuiu efetivamente para os fortes níveis sonoros encontrados no modo de manipulação brusca, além de o período de uso desses equipamentos variar entre 15 e 18 anos.

Observamos que o grupo C possuía uma plataforma constituída por peças plásticas e, em três das quatro incubadoras do grupo, a plataforma era manuseada a partir da rotação de uma peça que se encontrava na parte exterior da incubadora. Esse fato pode ter contribuído para que esse grupo apresentasse o menor NPS nessa situação, tanto no modo cuidadoso, quanto no brusco. As incubadoras do grupo D também possuíam peças plásticas na plataforma, mas, como o seu suporte era metálico, aumentou o nível de ruído produzido no modo cuidadoso, em comparação ao grupo $\mathrm{C}$.

Um estudo obteve $118 \mathrm{~dB}$ na elevação do leito e colchão da incubadora no modo cuidadoso e $122 \mathrm{~dB}$ no modo brusco, ambos em pico; ao abaixá-los, no modo brusco, o nível foi de 120 dB em pico ${ }^{(11)}$. Outra pesquisa constatou, no modo suave, $84,5 \mathrm{~dB}$ NPS, ao elevar 0 leito da incubadora, e, no modo brusco, 87,8 dB NPS; ao abaixá-lo, suavemente, gerou 83,6 dB NPS e, no modo brusco, 90,6 dB NPS ${ }^{(12)}$.

As situações de retirar e colocar a plataforma de base só foram mensuradas no grupo $A$, por ser este o único grupo em que a porta de cuidados intensivos mantinha-se aberta e travada na posição horizontal, permitindo, portanto, que se realizasse esse procedimento. No grupo $A$, as travas da porta de cuidados intensivos são botões de apertar, e estes, no modo brusco de manipulação, geram fortes NPSs, devido ao fato de ser mais cômodo fechar essa porta empurrando-a. Já nos grupos $\mathrm{C}$ e $\mathrm{D}$, as travas são botões de girar, o que causam um menor nível de ruído, pois a porta só fecha girando os botões.

A situação de retirar e colocar a plataforma de base deixa em evidência que a manipulação no modo brusco gera NPS muito mais intenso que no modo cuidadoso, portanto manipulá-la com cuidado é um meio efetivo de reduzir o ruído de impacto na incubadora.

As incubadoras dos quatro grupos, independentemente do período de uso, apresentaram resultados semelhantes na situação de elevar e abaixar a cúpula, havendo a necessidade de maior cuidado na realização dessa manipulação, pois esta, por si só, gera um NPS muito intenso. Estudo realizado num hospital geral do norte do Paraná mensurou 88,5 dB NPS ao elevar a cúpula no modo suave e $97 \mathrm{~dB}$ NPS no modo brusco; abaixá-la gerou 80,5 dB NPS e 92 dB NPS, nos modos suave e brusco, respectivamente ${ }^{(12)}$.

Podemos observar que, em todos os grupos, durante a situação de fechar as portas do gabinete inferior no modo de manipulação brusco, independente do período de uso dos equipamentos, foi produzido quase o triplo do nível de ruído que o da mesma situação realizada no modo cuidadoso. Mesmo o gabinete sendo localizado na parte inferior do suporte da incubadora e não fazendo parte efetiva do seu micro-ambiente, o nível de ruído produzido dentro do compartimento do RN é intenso, provavelmente pela ação da reverberação nesse ambiente totalmente metálico. Estudo semelhante encontrou $109 \mathrm{~dB}$, em pico, durante 0 ato de fechar normalmente as portas do gabinete inferior ${ }^{(11)}$.

$\mathrm{O}$ ato de tamborilar sobre a cúpula de acrílico gerou, no modo cuidadoso, níveis de ruído que variaram de 76,1 dB/C NPS a 93,9 dB/C NPS, níveis efetivamente intensos. Outro estudo mensurou um nível de ruído de 81 dB NPS durante o ato de tamborilar sobre a cúpula de acrílico da incubadora ${ }^{(12)}$. Essa situação que é vista corriqueiramente nos berçários, produz um nível de ruído de impacto elevado, motivo pelo qual deveria ser abolida.

Ao se colocar a prancheta sobre a cúpula, no modo brusco, com as portinholas fechadas, obteve-se $109 \mathrm{~dB}$, em pico ${ }^{(11)}$. Pesquisadores citam níveis sonoros de 80 a $89 \mathrm{~dB} / \mathrm{A} \mathrm{NPS}^{(13)}$. Na instituição pesquisada, o prontuário é uma pasta plástica, fato este que ajuda a minimizar o ruído quando ele é colocado de forma cuidadosa. Durante a coleta dos dados, em nove incubadoras pertencentes aos diferentes grupos, colocar o prontuário no modo cuidadoso não aumentou o NPS dentro do compartimento.

$O$ ato de colocar objetos sobre a cúpula da incubadora gerou, no modo suave, $78 \mathrm{~dB}$ NPS e, no modo brusco, 95,6 dB NPS, ambos em pico ${ }^{(12)}$. Outro estudo verificou que a colocação da mamadeira, sobre a cúpula, com cuidado, não gerou ruído algum, enquanto que, de modo brusco, o nível atingiu $116 \mathrm{~dB}$, em pico, com as portinholas fechadas $^{(11)}$.

Podemos notar que, nas situações de tamborilar e colocar objetos sobre a cúpula da incubadora, o nível de ruído produzido é elevado (pois a incubadora está totalmente fechada), sendo ainda mais intenso no modo de manipulação brusca. Isso pode ser explicado devido ao fenômeno da reverberação que ocorre nos espaços fechados, aumentando, assim, a duração do ruído de impacto ${ }^{(16)}$. Daí, a importância de se evitar colocar objetos em cima da cúpula da incubadora; quando isso for inevitável, devemos nos valer de estratégias que atenuam o seu impacto, como, por exemplo, a colocação 
O ruído gerado durante a manipulação...

Rodarte MDO, Scochi CGS, Leite AM, Fujinaga Cl, Zamberlan NE, Castral TC.

de um tecido flanelado/acolchoado sobre o acrílico da cúpula que, por sua vez, também reduz a intensidade direta da luz ambiente no interior da incubadora ${ }^{(2,14)}$.

Ressaltamos que, mesmo estando as rodinhas da incubadora bem distantes do compartimento do RN, o seu movimento produz nível efetivo de ruído para o bebê. Portanto, a manutenção periódica (com providências simples, como lubrificá-las) e o travamento destas, quando a incubadora não necessita ser deslocada, são formas eficazes para atenuar os ruídos produzidos durante o deslocamento do equipamento. $\mathrm{Na}$ instituição pesquisada, observamos que muitas incubadoras ficam com as rodinhas destravadas, gerando ruído adicional quando os profissionais manipulam as portinholas.

\section{CONCLUSÃO}

As médias encontradas entre os diferentes grupos de incubadoras, durante as situações de manipulação, ocorreram, provavelmente, tanto devido ao modelo do equipamento como ao tempo de uso, sendo o ruído menor naquelas mais novas. Detalhes técnicos nos equipamentos dos grupos $\mathrm{C}$ e $\mathrm{D}$, como uso de borrachas mais macias para amenizar o impacto do acrílico, de travas deslizantes ou giratórias para abrir e fechar as portinholas e porta de cuidados intensivos das incubadoras foram relevantes para amenizar o ruído; enquanto travas de pressão (tipo "apertar") mais antigas geraram NPSs superiores nas situações de manipulação (grupos A e B).

As situações que desencadearam maiores NPSs foram: fechar as portinholas e a porta de cuidados intensivos e abaixar a cúpula. Colocar o frasco de leite e o prontuário sobre a cúpula de acrílico da incubadora foram as situações que produziram menores NPSs no modo cuidadoso, entretanto essas mesmas situações apresentaram as maiores diferenças entre o modo de manipulação brusco e cuidadoso (17,1 dB/C ao se colocar o frasco de leite e 19,2 $\mathrm{dB} / \mathrm{C}$ ao se colocar o prontuário). $O$ modo de manipulação cuidadoso é um meio efetivo para minimizar os níveis sonoros produzidos durante as constantes situações de manipulação.

Podemos observar várias vezes, nos diferentes grupos, que mesmo a manipulação cuidadosa gerou um forte nível de ruído e que ele quase sempre dobra ou triplica o NPS quando a mesma situação é realizada bruscamente. Em algumas situações chegamos a ter a intensidade sonora quadruplicada, o que nos faz refletir sobre a relevância da manipulação cuidadosa para a redução dos NPSs gerados durante as constantes e corriqueiras situações de manipulação da incubadora.

Mesmo não tendo uma norma específica delimitando os níveis de ruído de impacto na incubadora, podemos afirmar que tais ruídos gerados durante as diferentes situações de manipulação são
Rev Latino-am Enfermagem 2005 janeiro-fevereiro; 13(1):79-85 www.eerp.usp.br/rlae

efetivamente fortes, mesmos nos grupos em que os NPSs foram inferiores. A manipulação cuidadosa, juntamente com um menor tempo de uso da incubadora, mostraram-se eficazes na redução do nível de intensidade sonora produzida durante o manuseio do equipamento. A manutenção preventiva e a mensuração objetiva e constante dos níveis de ruído das incubadoras poderiam ser um meio de se evitar que o RN utilizasse, por vários dias ou até meses, um equipamento com níveis excessivos de intensidade sonora, prevenindo-Ihe não só problemas auditivos, mas diversas respostas fisiológicas e comportamentais negativas, decorrentes da exposição ao ruído.

Levando em conta a imaturidade global dos RNs prematuros e a sua permanência por longos períodos nas incubadoras, consideramos que os níveis de ruído nas incubadoras mais seguro para a prevenção de danos auditivos seriam de acordo com o conforto acústico em hospitais, variando entre 35 e $45 \mathrm{~dB} /$ A NPS $^{(11)}$. Em relação aos ruídos de impacto, como ainda não há normas específicas que delimitem aqueles seguros para a audição dos bebês, ressaltamos que a manipulação cuidadosa da incubadora, juntamente com uma manutenção periódica e menor tempo de uso do equipamento, seriam formas relativamente eficazes para a redução dos NPSs durante a manipulação constante da incubadora.

Vimos que a manipulação cuidadosa da incubadora é um meio eficaz para minimizar os NPSs gerados durante as diversas situações de manipulação e, assim, destacamos a importância da educação continuada da equipe neonatal, abordando conteúdos sobre o ruído ambiente, em especial o controle e a manipulação cuidadosa de incubadoras durante a realização de procedimentos de apoio ao diagnóstico e terapêuticos. Destacamos aí o papel da enfermagem por assumir parcela significativa dos cuidados diretos e contínuos dos recémnascidos, manipulando constantemente as incubadoras, e, portanto, influenciando diretamente na redução ou aumento do ruído ambiente. A organização desse trabalho deve contemplar, ainda, a redução dos sons das vozes, monitores, alarmes, rádios, entre outros, sinalizando a UTIN como área de silêncio ${ }^{(2)}$.

A enfermagem tradicionalmente tem assumido a responsabilidade pelo controle do ambiente das unidades neonatais ${ }^{(2)}$, incluindo providências acerca da manutenção dos equipamentos da unidade. Com a sobrecarga dessas funções, torna-se relevante a inserção do fonoaudiólogo na equipe multiprofissional das unidades neonatais, implementando, também, ações preventivas, como o controle do ruído ambiente e a triagem auditiva neonatal, no intuito de promover a saúde auditiva do RNs e dos trabalhadores e manter um vínculo com a equipe do setor de manutenção, auxiliando no processo de aquisição dos equipamentos hospitalares, dentre outras atuações.

Percebemos que as unidades neonatais desse hospital-escola ainda contam com várias incubadoras de modelos antigos, cujo funcionamento e manipulação têm gerado ruídos, especialmente durante 
a manipulação das portinholas. Acrescentamos, ainda, o trabalho aí realizado, que inclui a inserção ativa dos pais no cuidado do filho, mesmo com o bebê em incubadora ${ }^{(19)}$. Nesse contexto, ressaltamos novamente a manipulação cuidadosa da incubadora como um meio efetivo de se minimizar os NPSs produzidos durante as diferentes situações de manipulação.

Concluímos este estudo esperando que os resultados instrumentalizem transformações na prática assistencial em unidades neonatais, subsidiando os programas de conscientização dos

\section{REFERÊNCIAS BIBLIOGRÁFICAS}

1. Tamez RN, Silva MJP. Impacto do ambiente da UTI neonatal no desenvolvimento neuromotor. In: Tamez RN, Silva MJP. Enfermagem na UTI neonatal: assistência ao recém-nascido de alto risco. Rio de Janeiro (RJ): Guanabara Koogan; 1999.

2. Scochi CGS, Riul MJS, Garcia CFD, Barradas LS, Pileggi So.Cuidado individualizado ao pequeno prematuro: o ambiente sensorial em unidade de terapia intensiva neonatal. Acta Paul Enfermagem, 2001 jan-abr; 14(1):9-16.

3. Marrese AM. El ambiente de la UCI neonatal y su influencia en el desarrollo del prematuro: un desafío para enfermería. Med Perinat Neonat 1996 jul-ago; 1(1):15-21.

4. Fasolo MI, Moreira RN, Abatti PJ. Avaliação de nível de ruído em incubadora. Pediatria 1994; 70(3):157-62.

5. Russo ICP. Ruídos, seus efeitos e medidas preventivas. In: Russo ICP. Acústica e psicoacústica aplicadas a fonoaudiologia. São Paulo (SP): Lovise; 1993.

6. Lichtig I. Avaliação audiológica do recém-nascido. In Kudo AM, Marcondes E, Lin L. Moriyama LT, Guimarães MLLG, et al. Fisioterapia, fonoaudiologia e terapia ocupacional em pediatria. São Paulo (SP): Sarvier; 1990.

7. American Academy of Pediatrics. Committee on environmental health. Noise: a hazard for the fetus an newborn. Pediatrics 1997 Oct.; 100(4):724-7.

8. Parrado MES, Costa AO Filho. O berçário de alto risco e o ruído das incubadoras. Rev Pró-Fono; 1992; 4(1):31-4.

9. Associação Brasileira de Normas Técnicas - ABNT(BR). NBR IEC 6012 19: equipamento eletromédico - parte 2: prescrições particulares para segurança de incubadoras de recém-nascido (RN). Rio de Janeiro (RJ): ABNT; 1997.

10. Weibley TT. Inside the incubator. MCN Am J Maternal Child Nurs 1989 Mar/Apr; 14(2):96-100.

11. Lichtig I, Maki K. Estudos de níveis de ruídos ambientais e de ruídos gerados pelas incubadoras em uma unidade de terapia intensiva neonatal. Pediatria 1992; 14(1):30-4.

12. Gomes CF, Crivari MMF. Os ruídos hospitalares e a audição do bebê. Rev Bras Otorrinolaringol 1998 set/out; 64(5):453-7.

13. Tamagna A, Veit ALH, Trindade DM. Avaliação do nível de ruído em ambiente hospitalar. Anais do $7^{\circ}$ Congresso de Biomecânica. [on line]. Disponível em: http://www.unicamp.br/fef/eventos/viicbb/068/ 705x.htm [acessado em: 05 set. 2001] profissionais da área de saúde, para o manejo cuidadoso da incubadora e a implantação de normas e protocolos de cuidado, com vistas ao controle e redução do ruído ambiental e das incubadoras, contribuindo para a melhoria da assistência e qualidade de vida dos RNs que necessitam dessa tecnologia para os cuidados especiais a eles destinados. Implementar essas ações dirigidas à equipe de saúde e clientela da unidade neonatal (pais e visitantes) e avaliar o seu impacto na redução do ruído em incubadoras e no processo de cuidar constituem motivações para a realização de estudos posteriores.

14. Consentino A, Malerba MC. Intervenciones reguladas en el cuidado del recién nacido prematuro extremo: protocolo de intervención mínima. Temas de Enfermeria Actualizadas 1996 jul/ago; 4(18):22-5.

15. Guimarães H, Oliveira AM, Spratley J, Mateus M, Orey C, Coelho $\mathrm{JL}$, et al. Le bruit dans une unité de soins intensifs néonatals. Arch Pédiatr 1996 nov; 3(11):1065-8.

16. Royster JD. Noise-induced hearing loss. In: Northen JL. Hearing disorders. Boston: Allyn and Baco; 1996. p. 177-88.

17. Leis, Decretos (BR). Segurança e medicina do trabalho. São Paulo (SP): Atlas; 1997.

18. Menegotto IH, Couto CM. Tópicos de acústica e psicoacústica relevantes em audiologia. In: Frota S. Fundamentos em fonoaudiologia: audiologia. Rio de Janeiro (RJ): Guanabara Koogan; 1998.

19. Scochi CGS, Kokuday MLP, Riul MJS, Rossanez, LSS, Fonseca, LMM, Leite AM. Incentivando o vínculo mãe-filho em situação de prematuridade: as intervenções de enfermagem no Hospital de Clínicas de Ribeirão Preto. Rev Latino-am Enfermagem 2003 jul/ago; 11(4):5315. 